\title{
Pflanzen der High Society: \\ Strelitzia reginae, eine Staude zu Ehren einer Königin von England
}

\author{
Peter Schubert \& Hilke Steinecke
}

\begin{abstract}
The crane flower or bird of paradise flower (Strelitzia reginae, Strelitziaceae) commemorates Charlotte of MecklenburgStrelitz. The botanical features and culturing of this plant are sketched. CHARLOTTE's life and her love for plants are briefly outlined. A particular cultivar of this flower is the lemon-yellow 'Mandela's Gold'. In 1996, it was presented by the South African embassy as a gift to the Palmengarten. Thus, for the first time it was publically displayed in a German botanical garden.
\end{abstract}

\section{Zusammenfassung}

Die Paradiesvogel- oder Kranichblume (Strelitzia reginae, Strelitziaceae) wurde zu Ehren von Charlotte von MecklenburgStrelitz benannt. Botanik und Kultur der Pflanze werden kurz vorgestellt. Zudem wird Charlottes Leben und ihre Liebe zur Botanik hervorgehoben. Eine besondere Sorte der Strelitzie mit zitronengelben Blüten ist 'Mandela's Gold'. Sie wurde 1996 von der Südafrikanischen Botschaft dem Palmengarten geschenkt und war damals erstmals in einem deutschen botanischen Garten zu sehen.

\section{Botanisches}

Die Paradiesvogelblume (Strelitzia reginae, Strelitziaceae) stammt aus der Kap-Region in Südafrika. Es handelt sich um eine immergrüne, bis etwa $2 \mathrm{~m}$ hohe Staude mit derben bis lederigen Blättern. Die langgestielten Blütenstände erinnern an den Kopf eines kranichähnlichen Vogels, worauf sich der Name Paradiesvogelblume bezieht. Bis zu acht Blüten werden von einem rötlichgrünen, derben und kahnoder schnabelförmigen Hochblatt umgeben. Die äußeren drei Blütenblätter sind orange bis gelb gefärbt. Die inneren schmalen, blauen Blütenblätter bilden ein spießförmiges Organ, das Griffel und Staubblätter umschließt.

Paradiesvogelblumen werden von Vögeln bestäubt. Sie setzen sich auf das Hochblatt, um Nektar zu trinken, wobei sie mit ihrem Gewicht auch den „Blauen Spieß“ herunterdrücken. Die zwei blauen Leisten klappen zur Seite, so dass die langen, schmalen Pollensäcke freigelegt werden. Sie entlassen klebrigen Pollen, der meist an den Füßen oder am Kopf der Vögel hängen bleibt. Bei einem nachfolgenden Besuch einer anderen Strelitzien-Blüte wird der Pollen effektiv übertragen.

Wegen ihrer langen Blütezeit sind Strelitzien ideale Schnittblumen. Bei uns werden sie üblicherweise als Kübelpflanzen gehalten. Im Sommer mögen sie einen Standort im Freien und vertragen Licht und Sonne. Die Paradiesvogelblume kann in ganz normaler Gartenerde gehalten werden, eine Mischung zu gleichen Teilen aus Gartenerde, Laub und Kies ist ebenso geeignet. Im Sommer, während der Wachstumsphase, sollte regelmäßig Flüssigdünger gegeben werden. Wenn der Topf zu eng wird, können von außen einzelne Blätter entfernt werden. Überwintert wird an einem hellen, nicht zu warmen Standort bei ca. 12-15 Grad. Für einen Blütenansatz ist es wichtig, der Pflanze eine Ruhepause zu gönnen, in der sie nur sehr sparsam gegossen und nicht gedüngt wird.

\section{Benannt nach einer Königin}

Exotisch, sehr elegant, wenn auch etwas steif, wirken die Blütenstände der Pflanze mit dem botanischen Namen Strelitzia reginae. Übersetzt bedeutet der Name „die Strelitzia der Königin“. Ein ungewöhnlicher Name, doch angesichts ihrer Schönheit sehr treffend für eine Pflanze, die ihren Namen einer englischen Königin verdankt: Sophie Charlotte von England aus dem Hause Mecklenburg-Strelitz (1744-1818).

\section{Von einer Prinzessin zur Königin von England}

Geboren in Mirow in Mecklenburg, genoss Prinzessin Sophie Charlotte eine strenge 


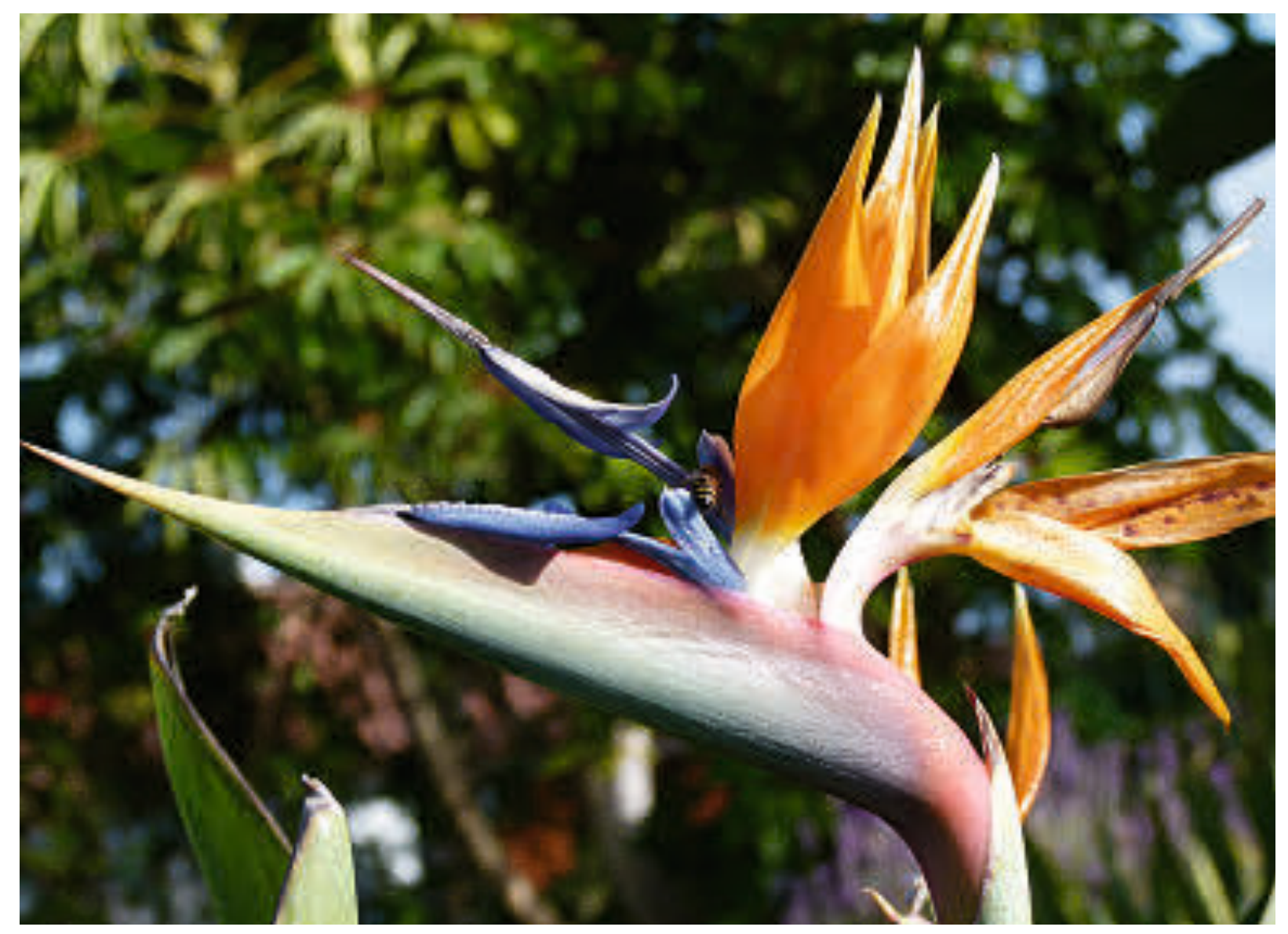

musische, literarische und hauswirtschaftliche Erziehung. Der Hauslehrer der Familie, der lutherische Theologe Gentzmer, unterwies sie in Religion, aber auch in Naturphilosophie, Mineralogie und Botanik und traf damit bereits bei der jungen Sophie Charlotte auf großes Interesse. Ein couragierter Brief an den preußischen König, in dem sie sich über das Betragen der preußischen Armee während des 7-jährigen Krieges in Mecklenburg beschwert hat, soll den englischen König veranlasst haben, ihr einen Heiratsantrag zu machen. Sie war erst 17 Jahre alt, als sie am 8. September 1761 den 22-jährigen König Georg III. von England heiratete und mit der Krönung am 22. September desselben Jahres zur Queen Charlotte, Königin von Großbritannien und Irland wurde. Als privaten Rückzugsort kaufte König Georg III. seiner jungen Braut Buckingham House, das dementsprechend nach seiner Renovierung

Abb. 1: Blühende Strelitzie auf Teneriffa.
Queen's House genannt wurde. Erst sechzig Jahre später, nach umfangreichen Um- und Erweiterungsbauten, erhielt das Gebäude den $\mathrm{Na}$ men Buckingham zurück. Heute ist Buckingham Palace bekanntlich Residenz der amtierenden Königin Elisabeth II.

\section{Charlotte und die Botanik}

König Georg III. liebte das Landleben. Spaßeshalber wurde er deshalb gelegentlich auch „Farmer Georg“ genannt. Dieser Passion war auch Charlotte recht zugetan. Angesichts ähnlicher Interessen führte das Paar eine glückliche Ehe. Aus ihr gingen 15 Kinder hervor, 9 Söhne und 6 Töchter. Aus der Politik hielt sich Sophie Charlotte heraus. Sie widmete sich vorwiegend häuslichen Aufgaben. Damals nicht üblich, kümmerte sie sich als Königin selbst um die Erziehung ihrer Kinder. Auch zeigte sie sich sehr großzügig gegen Hilfsbedürftigen und war daher in der Bevölkerung sehr beliebt. Ohne große Schlagzeilen lebte die Familie relativ bescheiden im Kew Palace. Ihr 


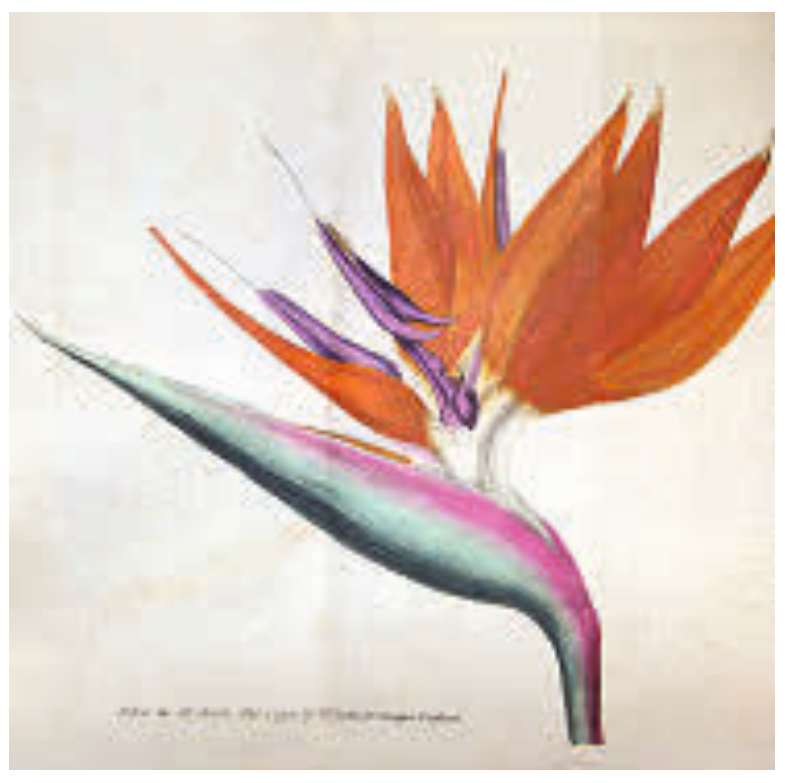

Interesse galt weniger dem Haus als dem großen Landschaftspark, der das Schloss umgab. Hatte sich dieser bereits unter der Schwiegermutter Marie Charlottes allmählich vom Lustgarten zum botanischen Garten gewandelt, kamen unter der Herrschaft GeORG III. vermehrt neue Pflanzen hinzu. Mit William Aiton fand der König einen hervorragenden Gärtner und Botaniker und Vorsteher seines Gartens. Und mit Sir Joseph Banks, der Mitte des 18. Jahrhunderts James Cook auf seiner ersten Reise und Weltumsegelung (1768-1771) begleitet hatte, gewann er einen inoffiziellen Direktor der Königlichen Gärten. Als Präsident der Royal Society und Naturforscher genoss er in der damaligen Welt höchstes Ansehen. Vor allem BANks' Verdienst war es, der, um die Gärten weiter auszubauen, sogenannte Pflanzenjäger ausschickte. Diese sollten in den neuen Kolonien des Empires noch nicht entdeckte Pflanzen sammeln. Damit legte er den Grundstein für Kew Gardens, der heute größten botanischen Pflanzensammlung der Welt. Wie groß auch Charlottes Interesse am Garten und seinen Pflanzen war, zeigt, dass sie zusammen mit ihren Töchtern bei Sir James Edward Sмiтh, dem ersten Präsidenten der renommierten Londoner LinNé-Gesellschaft, BotanikUnterricht nahm. Ihre Leidenschaft war so auffällig, dass man sie im Volk liebevoll als „Queen of Botany" titulierte.

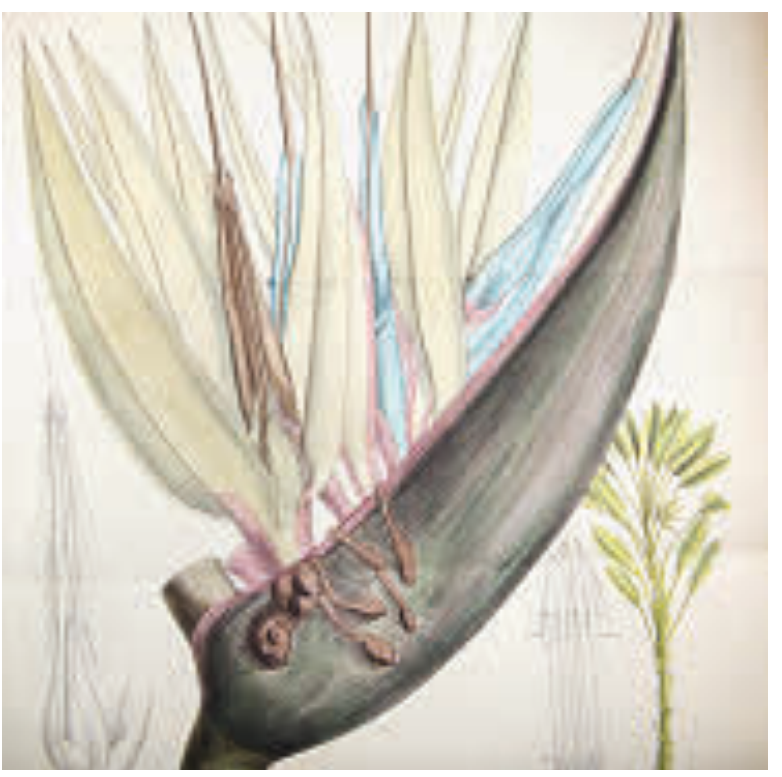

Als die Geisteskrankheit ihres Gattens erschreckende Ausmaße zeigte, erwarb sie 1790 Frogmore House, ein Palais, das eine halbe Meile südwestlich von Schloss Windsor liegt. Sie nannte es ihr kleines Paradies, weil sie dort mit ihren Töchtern botanischen Studien nachgehen konnte, ohne die unmittelbare Belastung, die von der Krankheit ihres Mannes ausging.

\section{Die Strelitzie, eine neu entdeckte und attraktive Südafrikanerin}

Als der Schotte Francis Masson (1741-1805), der erste offizielle Pflanzenjäger der Königlichen Gärten von Kew und Begleiter von Kapitän James Cook auf seiner zweiten Weltumsegelung, 1773 in Küstenwäldern und an Flussufern der östlichen Kap-Provinz eine attraktive Staude entdeckte, wurde sie zunächst zu der erst zwei Jahre vorher beschriebenen Gattung Heliconia gestellt. Als sich jedoch große Unterschiede $\mathrm{zu}$ dieser Gattung zeigten, schlug angesichts der Schönheit der Pflanze und der Leidenschaft Charlottes für Pflanzen Sir Joseph Banks vor, ihr den Namen sei-

Abb. 2 (links): Blütenstand einer Strelitzie, abgebildet im Curtis‘ Botanical Magazine Nr. 119.

Abb. 3 (rechts): Strelitzia nicolai, abgebildet im Curtis Botanical Magazine Nr. 7098. 
ner Königin zu geben. Im Jahr 1789 wurde von William Aiton der Name Strelitzia reginae in der Zeitschrift Hortus Kewensis gültig veröffentlicht. Im Curtis's Botanical Magazine, einer Zeitschrift, in der Pflanzenneuheiten aus den Königlichen Gärten vorgestellt wurden, erschien bereits 1790 eine ausführliche Beschreibung der neu entdeckten Pflanze mit Kulturhinweisen und einer besonders prächtigen und großen Illustration ihres Blütenstandes. Nach ihrer Entdeckung wurden weitere verwandte Arten der Strelitzia reginae entdeckt. So tragen noch 4 weitere Arten den Namen des Elternhauses von Königin Charlotte. Bei einer davon, Strelitzia nicolai, die über $5 \mathrm{~m}$ hoch wird, nimmt der Artname Bezug auf Zar Nikolaus I. von Russland, womit zwei europäische Adelshäuser in einer Pflanze vereint sind. Auch wurde nur wenige Jahre nach der "normalen“ Strelitzia 1796 eine Varietät (var. juncea) mit runden Blattstielen und reduzierter Blattspreite gefunden, die gleichfalls sehr schön ist (Musgrave et al. 1999).

Charlotte war vermutlich stolz auf ihre Blume. 1817, ein Jahr vor ihrem Tod, schickte sie ein Exemplar im Kübel in ihre Heimat Mecklenburg, wo sie überhaupt das erste Mal in Deutschland zu sehen war. Zuerst in Hohenzieritz, dann in der fürstlichen Orangerie in Neustrelitz untergebracht, zeigte sie 1822 die ersten Blüten, leider erst drei Jahre nach CHARLOTTES Tod. Noch heute erinnert man sich in Mecklenburg gerne an Charlotte. Man ist stolz, dass eine englische Königin aus Mecklenburg stammt. Die Stadt Neustrelitz hat 1995 die Strelitzie als Stadtblume auserwählt. Sie fehlt seitdem bei keiner Stadtfeierlichkeit und wird zu offiziellen Anlässen verschenkt. Ein Strelitzienfest wurde aus der Taufe gehoben. Mit dabei ist immer das Stadtmaskottchen Strelitzius, das auf einem stilisierten Blütenstand ein verschmitzes Gesicht zeigt (Jipp 1995).

Abb. 4 (oben): Strelitzien im Botanischen Garten von Kirstenbosch/Südafrika.

Abb. 5 (unten): Strelitzia nicolai in einem Garten auf Teneriffa.
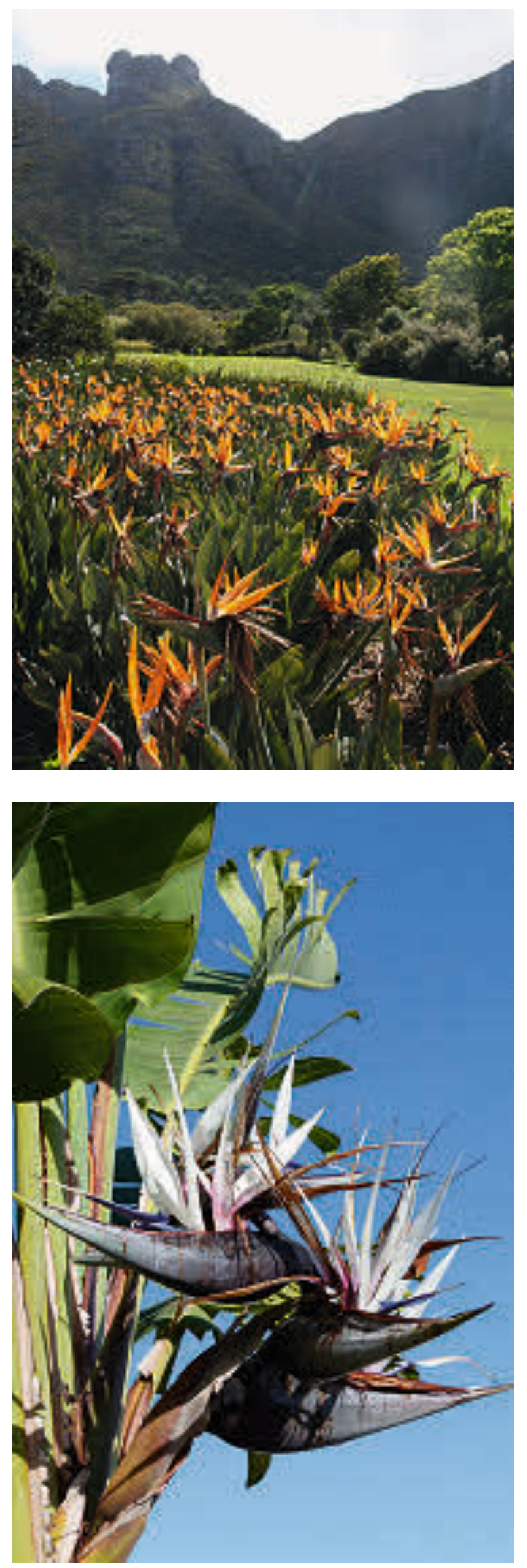


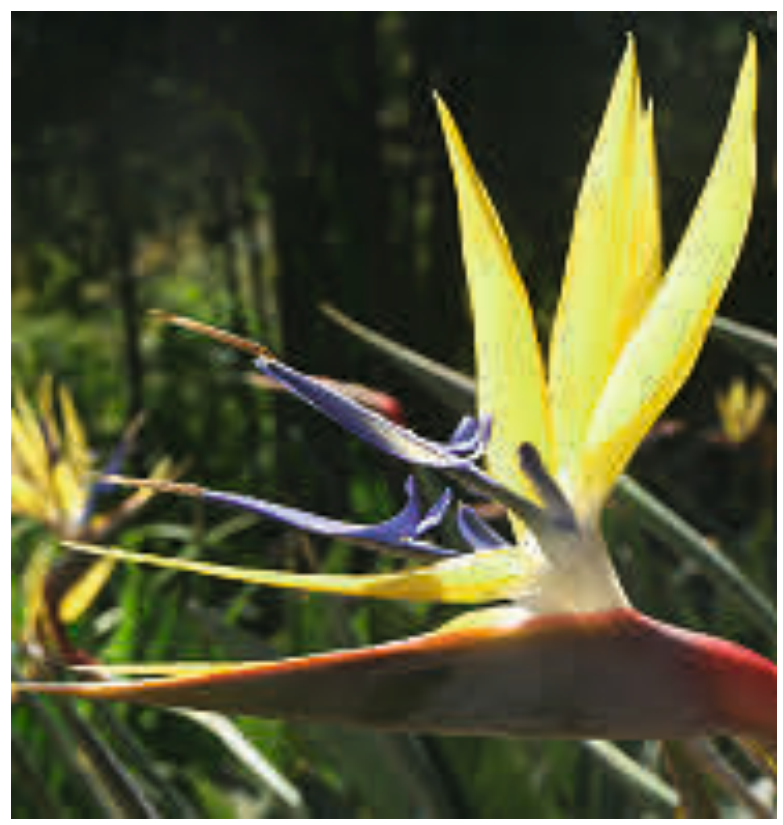

\section{Strelitzien, auch heute sehr beliebt}

Aber nicht nur in Mecklenburg schmückt man sich mit der Strelitzie. Auch in Los Angeles gilt sie als Blume der Stadt, wohl deshalb, weil die Blütenstängel mit ihren Blüten von leuchtend gelber und blauer Farbe so exotisch aussehen. Sie lassen die Vorstellung eines Paradiesvogels aufkommen, weshalb die Strelitzie oft auch als Paradiesvogelblume bezeichnet wird. Es gibt kaum einen tropischen Garten, in dem sie nicht zu finden ist. Dort, wo sie nicht im Freien wachsen kann, wird sie als Kübelpflanze gehalten. Man muss heute kein Mitglied der High Society sein und über geräumige Orangerien verfügen, um sie kultivieren zu können. In einem kleinen Wintergarten oder sogar in der Wohnung auf der Fensterbank lässt sich die Paradiesvogelblume bei guter Pflege durchaus erfolgreich kultivieren. Und wer das nicht möchte, an ihr aber trotzdem Gefallen findet, kann sie als lange haltbare Schnittblume erwerben. Jedes Jahr kaufen deshalb abertausende Kanaren- und Madeira-Urlauber gut verpackt in Kisten die Blütenstängel der Strelitzie als hübsches Urlaubssouvenir. Auch gibt es heute verschiedene Sorten: kleinblütige, großblütige, solche mit abweichender Blütenform und -farbe. Sie stammen von Pflanzen, die Gärtner ausgelesen und anschließend vermehrt haben.

\section{7. 'Mandela's Gold'}

In den 1970er-Jahren traten im botanischen Garten Kirstenbosch wenige leuchtend gelb blühende Exemplare der Strelitze auf. Der damalige Kurator John Winter startete ein Projekt, durch gezielte Bestäubung und Auslese diese gelben Strelitzien zu vermehren und dabei auch bei den Nachkommen diese besondere Blütenfarbe dauerhaft zu erhalten. Nach zwanzig Jahre währender züchterischer Arbeit konnte die Sorte 'Kirstenbosch Gold' kommerziell angeboten werden. Im Jahr 1996 wurde die Sorte zu Ehren von Nelson Mandela 'Mandela's Gold' getauft. Somit hat die gelbblütige Strelitzie sogar einen Namensbezug zu zwei bedeutenden Persönlichkeiten: einer englischen Königin und dem ehemaligen führenden Anti-Apartheid-Kämpfer Südafrikas und erstem schwarzen Präsidenten des Landes (1994 - 1999). Ob dies allerdings Königin Charlotte, deren Gatte sehr konservativ dachte, recht gewesen wäre, mag bezweifelt werden. Wie auch immer, 'Mandela's Gold' wurde dem Frankfurter Palmengarten 1996 während eines aufwendigen Festaktes von der südafrikanischen Botschafterin übergeben und war damit erstmals in einem deutschen botanischen Garten zu sehen.

\section{Literatur}

Jipp, K.-E. 1995: Die Strelitzie und ihre abenteuerliche Geschichte. - Kiel.

Musgrave, T., Gardner, C. \& Musgrave, W. 1999: Pflanzensammler und -entdecker. Zweihundert Jahre abenteuerliche Expeditionen. - München. 